\title{
GRAPH BASED RECOGNITION OF GRID PATTERN IN STREET NETWORKS
}

\author{
Jing Tian ${ }^{\mathrm{a}, \mathrm{b}}$, Tinghua $\mathrm{Ai}^{\mathrm{a}, \mathrm{b}}$, Xiaobin Jia ${ }^{\text {a.b }}$ \\ ${ }^{a}$ School of Resource and Environment Science, Wuhan University, 129Luoyu Road, Wuhan, 430079,China-(yutaka- \\ 2010@163.com, tinghua_ai@tom.com, jiaxiaobin_123@126.com) \\ ${ }^{\mathrm{b}}$ Key Laboratory of Geographic Information System, Ministry of Education, Wuhan University, 129Luoyu Road, \\ Wuhan, 430079, China
}

KEY WORDS: Spatial information Sciences, Cartography, Generalization, Pattern, Networks, Vector

\begin{abstract}
:
Pattern recognition is an important step in map generalization. Pattern recognition in street network is significant for street network generalization. A grid is characterized by a set of mostly parallel lines, which are crossed by a second set of parallel lines with roughly right angle. Inspired by object recognition in image processing, this paper presents an approach to the grid recognition in street network based on graph theory. Firstly, the bridges and isolated points of the network are identified and deleted repeatedly. Secondly, the similar orientation graph is created, in which the vertices represent street segments and the edges represent the similar orientation relation between streets. Thirdly, the candidates are extracted through graph operators such as finding connected component, finding maximal complete sub-graph, join and intersection. Finally, the candidate are evaluated by deleting bridges and isolated lines repeatedly, reorganizing them into stroke models, changing these stroke models into street intersection graphs in which vertices represent strokes and edges represent strokes intersecting each other, and then calculating the clustering coefficient of these graphs. Experimental result shows the proposed approach is valid in detecting the grid pattern in lower degradation situation.
\end{abstract}

\section{INTRODUCTION}

Ideas of scale and pattern are central to the process of interpretation in the geosciences. The patterns that are evident at any given scale will have specific causes and consequences (Mackaness, 2007).

What is pattern and pattern recognition? In tradition, pattern recognition is the scientific discipline whose goal is the classification of objects into a number of categories or classes. Depending on the application, these objects can be images or signal waveforms or any types of measurements that need to be classified (Theodoridis and Koutroumbas, 2004). The "pattern" refers to objects. From the geosciences experts' view, patterns can be organized into partonomic hierarchies reflecting relations between parts and subparts (Mackness and Edwards, 2002). In summary, pattern can be an object, object cluster, property with an object or object cluster, and relation between objects.

Pattern recognition techniques have been applied to machine vision, character recognition, and computer-aided diagnosis. These applications use images as information source and benefit from recognition of specific pattern in images. In the raster mode, the main tasks of pattern recognition are object detection and feature extraction. Here, street must be extract from image and this work is the focus. In vector mode, however, the elementary objects points, lines and polygons already exist in spatial database. Here, streets already exist in geographical spatial database and the recognition of relations and structures among streets is our interest. Traditional pattern recognition pays little attention to the latter.

In recent years, pattern recognition of spatial cluster object has come to assume increasing importance in the map generalization field. The relation between pattern and map generalization is that pattern recognition is the start point of map generalization, and pattern maintaining is the aim of the map generalization. Maintaining patterns after generalization is not only the generalization guideline but also the generalization result.

Identifying and extracting the spatial patterns of street networks is significant for network generalization. Maintaining the pattern of the street network after generalization is the basic principle in selection of streets. The pattern here refers to the structure of street network. However, most street network generalization methods pay no attention to principle. Zhang (2004) emphasized "modeling the patterns as input to generalization algorithm”.

Perceptual organization refers to the ability of human beings to form meaningful groupings of low level tokens detected in an image (Sarkar and Boyer, 1994). Sarkar and Boyer (1994) present a hierarchical approach to perceptual organization for detecting structure among tokens. Tokens are perceptual objects which are to be organized. The tokens may be points, straight lines, curves, parallel lines and rectangle, etc. The tokens at each level have specific attributes, and then some relation graphs whose nodes represent tokens and edges represent the association among tokens is created using these attributes. For example, proximity graph is a graph whose links join nodes represent tokens having points close together. Tokens at high level are extracted from the tokens at low level by standardized graph operations.

Inspired by Sarkar and Boyer's idea (1994), this paper presents an approach to recognition of grid pattern in the street network. A grid is characterized by a set of roughly parallel lines, which are crossed by a second set of parallel lines with roughly perpendicular angle. A set of parallel lines consists of line segments having proximity and similar orientation. The parallel lines and the grid are extracted by graph theoretic operators such as finding connected component, finding maximal complete sub-graph, join and intersection.

* Jing Tian. e-mail: yutaka-2010@163.com; phone: 86-13628636229. 
The rest of this paper is organized as follows. Section 2 reviews related work on the recognition of patterns in street network. Section 3 introduced concepts and definitions in graph theory which are used in this paper. Section 4 presents the approach to the recognition of grid pattern in detail. An experiment is conducted in order to test the approach and advantages and disadvantages are discussed in Section 5. Finally, section 6 concludes the paper and offers several avenues for future work.

\section{RELATED WORK}

\subsection{Main patterns in street network}

Street networks consist of a large amount of streets which intersect with each other in different mode. The patterns can be classified according to different criterions. From geometric view, In Zhang's research (2004), discernable patterns in road networks are star-like, grid-like and irregular pattern. Marshall (2005) proposed that main patterns are linear, treelike, radial, cellular and hybrid forms. Heinzle et al (2006) suggested the typical patterns are strokes as type of linear forms, grids as type of cellular forms, star as type of radial forms and circular roads as type of cellular forms. It is important to note that the grid pattern in Heinzle et al (2005) only consists of streets which are parts of grid. The grid is defined in local sense. From topological view, there are three important patterns: regular, small-world and random (Watts and Strogatz, 1998).If criterion is the distribution of the node degrees in the network, the random and scale-free pattern can be used in such classification. Xie and Levinson (2007) defined four typical connection patterns in road networks. They are Ring and Web form in circuit networks and Star and Hub-and Spoke form in branching networks. Circuit is defined as a closed path that begins and ends at the same vertex. Branching networks consist of sets of connected lines without any complete circuit.

\subsection{Characteristics and Quantification Measures of Patterns}

A grid is characterized by a set of roughly parallel streets, which are crossed by a second set of parallel streets with roughly perpendicular angle. The main quantification measure is distribution of street direction from statistical view. A star is characterized by a "fuzzy center" degree from which streets radiate or converge. If the fuzzy center is clustered to one intersection, the connectivity degree of it is high compared with other intersections. A ring is approximate a circle. There are rings in different sizes: roundabouts, rings around the hub-like junction, rings surrounding a city center. The ring can be described by shape index: compactness, convexity, and geometric moments. To check whether a network is a smallworld, characteristic path length and clustering coefficient are proposed. For scale-free network, degree and degree distribution are used. The degree of a node is the number of edges incident with node. The degree distribution is defined as the probability of nodes with specific number of links. Porta et al (2006) found the clustering coefficient of grid pattern is extremely small. It indicates that the geometric pattern and topological pattern of street network have intrinsic relation.

\subsection{Methods of Recognition of Patterns}

For grid pattern, Heinzle et al (2005) select so called CRS nodes, at which four edges intersect, as starting points, investigate the histogram of the edge directions, and then examine the polygon regarding its similarity to the neighbor polygons. Yang et al (2010) proposed a method for identifying grid pattern. Their method first builds a node-edge topology, generates polygons from these edges, and then calculates a set of parameters, and finally identifies grid via a multi-criteria decision method. These two methods for grid recognition are both polygon-based structural recognition approach.

For star pattern, Heinzle et al $(2005,2007)$ take any node in the graph as potential center point, use the Dijkstra algorithm to compute the single source shortest path from this node to all other nodes, and then use a circle with a certain radius to search the center. The fuzzy center is determined by a cluster algorithm. The problem of this approach is, as they pointed out, deficiency of knowledge about the search radius and the range of the configurations.

For ring pattern, Heinzle et al (2006) use convex hull peeling techniques to determine the approximate position for the city center, neighbouring polygons are aggregated as long as they are not separated by a major stroke subsequently, and then some candidates are sought, the geometric moments curvature and convexity are used to evaluate the candidates. The problem of this approach is the huge uncertainty to evaluate whether the candidate is a ring.

It is easy to check whether a network is a small-world network. The small-world has a small characteristic path length as random graphs and a much larger coefficient than random graph. For scale-free networks, it is easy to check whether the degree distribution follows a power law.

\subsection{Summary}

We can conclude from analysis above: (1) There are much work on classification and qualitative description of geometric pattern in street network, but little work on how to quantify the characteristics and how to detect geometric patterns. (2) It is easy for people to recognition of geometric patterns cognitively and difficult to formalize them, whereas it is difficult for people to recognition of topological patterns and easy to formalize them. The association of various measurements has not been studied.

This paper proposes a method for detecting grid pattern. The major difference between our proposed method and the methods of Heinzle et al (2005) and Yang et al (2010) is that our method detects grid patterns by street segments grouping.

\section{CONCEPTS AND DEFINITION IN GRAPH THEORY}

Street network is usually modeled using a graph, where vertices represent intersections and edges represent street segments. This section introduces some basic graph concepts and definitions which will be used in the next two sections. For a more complete introduction to graph theory, readers can refer for example to Diestel (2006).

A graph of $G$ consists of a finite set of vertices (or nodes, or points) and a finite set of edges (or links, or lines). A graph is often denoted as $G(V, E)$ where $V=\left\{v_{1}, v_{2} \cdots v_{n}\right\}$ and $E=\left\{v_{i}, v_{j}\right\}$. Two vertices $v_{i}, v_{j}$ of $G$ are adjacent, if $V_{i} V_{j}$ is an edge of $G$.The number of vertices of a graph $G$ is its order. $G$ is undirected graph if $E$ is unordered set. A complete graph $G=(V, E)$ is one in which each vertex is connected to every other vertex. Let 
$G=(V, E)$ and $G^{\prime}=\left(V^{\prime}, E^{\prime}\right)$ be two graphs. If $V^{\prime} \subseteq V$ and $E^{\prime} \subseteq E$, then $G^{\prime}$ is a subgraph of $G$. A maximal complete subgraph is a complete subgraph of $G$ that contains as many vertices of $G$ as possible. A non-empty graph is called connected if any two of its vertices are linked by a path in $G$. A maximal connected subgraph of $G$ is called connected component of $G$.The degree of a vertex is the number of edges at this vertex. A vertex of degree 0 is isolated. A vertex of a connected graph is an articulation vertex if its removal would create a separate graph. An edge of a connected graph is a bridge if deleting it would create a separate graph. We set $G \cap G^{\prime}:=\left(V \cap V^{\prime}, E \cap E^{\prime}\right)$, where $\cap$ is intersection operation. Join is an operation between two graphs that linking every vertex of one graph to every vertex of the other graph.

A relation graph is a graph in which the vertices represent entities and the edges represent specific relationships among entities. The structural representation of a street network proposed by Jiang and Claramunt (2004) is a relation graph, where vertices represented named streets and edges represent intersection relation among streets. The street segment intersection graph is a graph whose vertices represented street segments and edges represent intersection relation among street segments. The similar orientation graph is a graph whose vertices represented street segments and edges represent similar orientation among street segments. The orientation of the street segment is defined by its azimuth angle, whose range is $\left[0^{\circ}\right.$, $180^{\circ}$ ). The range of angular separation between two street segments is $\left[0^{\circ}, 90^{\circ}\right]$. The street intersection graph is a graph whose vertices correspond to entire streets and edges correspond to intersection relation among entire streets. The entire street consists of street segments which satisfy "good continuation” rule (Thomson and Richardson, 1999).

\section{RECOGNITION OF GRID PATTERN IN STREET NETWORKS}

\subsection{Characterization of Grid}

If the element considered is line, a grid is characterized by a set of roughly parallel streets, which are crossed by a second set of parallel streets with roughly perpendicular angle. The perpendicular angle is no compelling characteristic of a grid. If the element considered is polygon, a grid is composed of a set of polygons with similar shape and connect mode. Similar to viewpoint of the Heinzle et al (2005) and Xie and Levinson (2007) , we define grid as a closed structure consist by a set of mostly parallel lines, which are crossed by a second set of parallel lines, not include other lines. Hence, for the recognition of a grid, the key questions arise: (1) How can we find the two sets of parallel lines? (2) How can we evaluate whether the result is grid or not?

In the perceptual organization, Sarkar and Boyer (1994) chose length of two lines, distance perpendicular to the smaller line from its midpoint to larger line and angular separation to describe parallelism. The value of these parameters' threshold is decided using statistical method and experiments. By formulating the perceptual characteristics leading to human recognition of parallelism in terms of several distinctive forces, Ip and Wong (1997) developed a force-driven model as a new optimization strategy to perform correspondence establishment between points in the matching curves. To detect parallel segments after coupling, they first make sure that the orientations fall within an allowable limit. From analysis above, we can conclude that the angular separation between two segments is the most important factor for recognition of parallelism.

Porta et al (2006) found the clustering coefficient of grid pattern is extremely small. It indicates that the grid pattern and clustering coefficient have intrinsic relation. In fact, transforming a perfect grid to street intersection graph (vertices represent entire streets and edges represent intersection relation), the value of clustering coefficient of this graph is 0 . The value in Porta et al's experiment is not 0 because there exists some disturb streets shown in Figure 1. The grid pattern referred by Porta et al (2006) is in global sense. The clustering coefficient can be used to evaluate the grid.

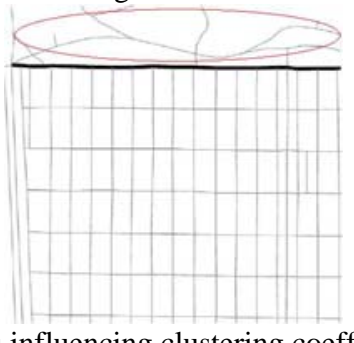

Figure 1. Streets influencing clustering coefficient (modified from Porta et al, 2006)

\subsection{The Approach to Recognition of Grid Pattern}

The core idea is that grids are composed of two groups of parallel streets. There exist relations among line segments in each group and among line segments in two groups. The angle of every line segment in the same group is similar, including the parallelism and collinearity. The line segments in one group is intersected at least one line segments in another group. Hence, we first take angular similarity as compelling condition. Through the graph-theoretic operations such as finding connected component, finding maximal complete subgraph, intersection and join, the candidates are sought. Finally, the street intersection graph is formed and clustering coefficient is computed to evaluate the candidates. The logic of this idea is that all parallel lines would be found and grouped through their angular separation first and the final result is the subset of these parallel line segments if a grid exists. The candidate is filter through graph-theoretic operations stage by stage. The steps are described in more detail in the following:

Step 1: Data preprocessing. The street network is organized as graph in which the vertices represent street intersections and edges represent street segments, i.e. primal graph. Inspired by Xie et al (2007), identify the bridges, delete bridges and isolated vertices until there are no bridges and isolated vertices in the graph. The reason is that a grid does not contain any bridges and isolated vertices. This step is to clean and refine the street network. Figure 2 shows a simple example. Deleting bridge 13 produces Figure2 (b), removing isolated vertex produces Figure2 (c), and Figure2 (d) is street segment intersection graph of Figure2(c). 


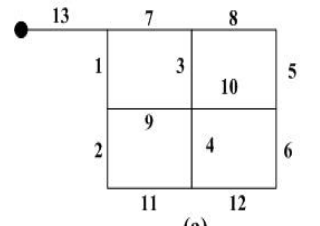

(a)

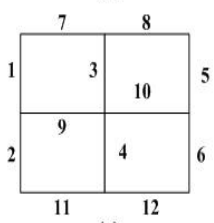

(c)

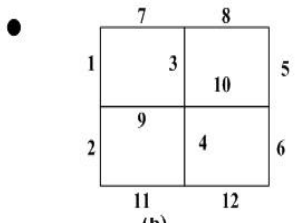

(b)

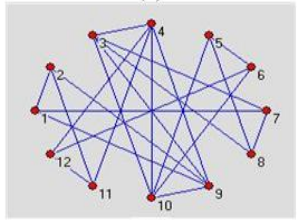

Figure 2. Simple Example ((d) visualization by Pajek) Step 2: Street grouping. The similar orientation graph is formed in which the vertices represent street segments and edges represent street segments having similar orientation. Figure 3 shows a similar orientation graph of Figure 2(c) with two connected component. There exist no grid or the angular separation is setting too large, if there are only one connected component in similar orientation graph. For example, if the angular separation is $90^{\circ}$, of course there is only one connected component. The intention of this step is that the street segments which are likely to form the grid are extracted, maybe including a lot of street segments disappear in final results.

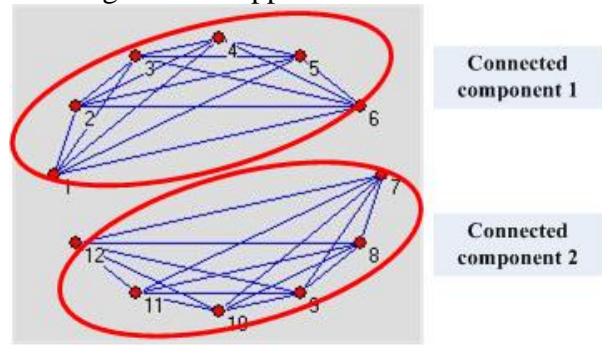

Figure 3. Similar orientation graph with two connected component (visualization by Pajek)

Step 3: Forming sets of parallel streets. For each connected component of similar orientation graph, their maximal complete subgraph is found. The idea of this step is that the orientation of every line segment in one set is similar to each other, including the parallelism and collinearity. This is based on the truth "line segments' angle in one group of parallel lines is similar to each other.” This step eliminates the streets which are not parts of a grid. Figure 4 shows the maximal complete graph of two connected component in Figure 3. The Maximal complete graph is the same as the connected component because the connected component self is complete graph. See section 5.1 for general case.
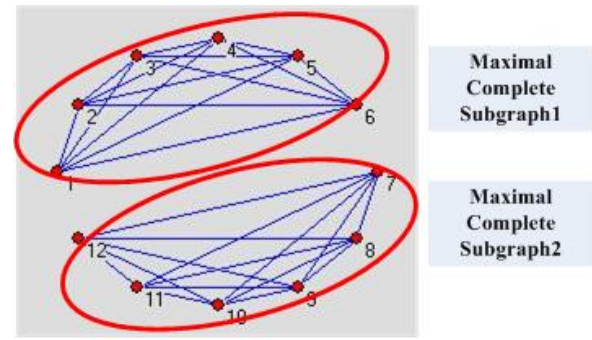

Figure 4. Maximal complete subgraph (visualization by Pajek) Step 4: Linking two sets of parallel streets to form candidates. Select two maximal complete subgraph, "Join" them, then "intersection" with the street segment intersection graph. Figure 5 shows the "Join" and "Intersection" operation.

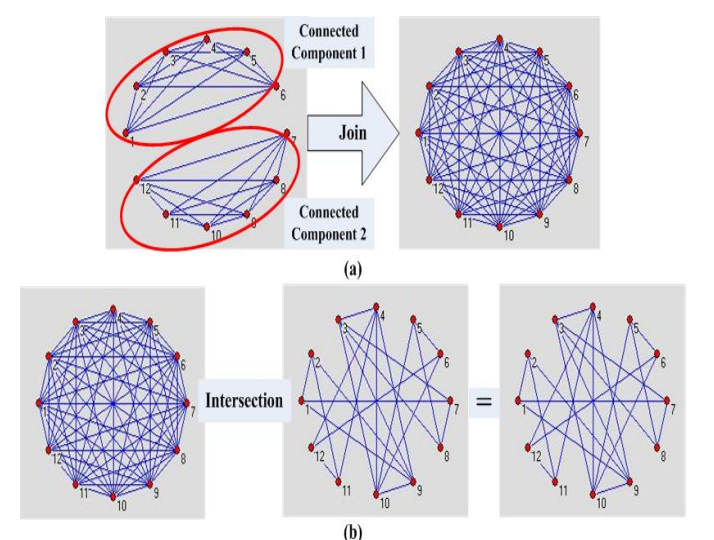

Figure 5. (a) “Join” and (b) “Intersection” (visualization by Pajek)

Step 5: Data post-processing. Delete the bridges and isolated vertices until there are no bridges and isolated vertices in the street network after processing by step 4 . This step considers the closed property of a grid. Figure 2 (c) shows a candidate of Figure 2(a). See Figure 10 and Figure 11 for a complex example.

Step 6: Evaluation. For each candidate, the street segments are linked to form entire streets using "good continuation" rule (Thomson et al 1999), then the street intersection graph is created. If the order of the graph is less than 6 , then delete the candidate. The reason is that the minimal grid contains 6 entire streets. The clustering coefficient of this graph is computed. The candidate is written out as final result if its clustering coefficient value is 0 . Figure 6(a) shows the entire street of Figure 2(c). Figure 6(b) shows the street intersection graph of Figure 6(a).

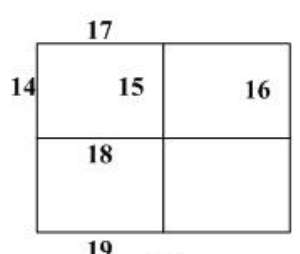

(a)

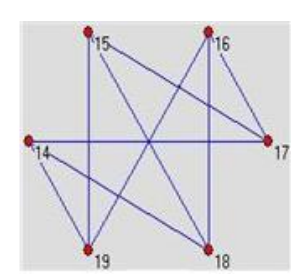

(b)
Figure 6. (a) Entire streets and (b) Street intersection graph (visualization by Pajek)

\section{EXPERIMENTS AND DISCUSSION}

\subsection{Experiments}

The experiments are conducted on Windows XP platform. The function of creating maximal complete sub-graph is performed using the Graph Theory Toolbox (Iglin, 2003) associated with Matlab (version 7.1). The clustering coefficient is computed using Pajek (Batagelj et al, 1997). Other functions are implemented in Visual C++ (Version 6.0) and integrated into a map generalization system named DoMap. The experiment data is digitized from Wang et al (1993) using ArcGIS (version 9.2). The experimental data is show in Figure 7. The number is the street segment label. 


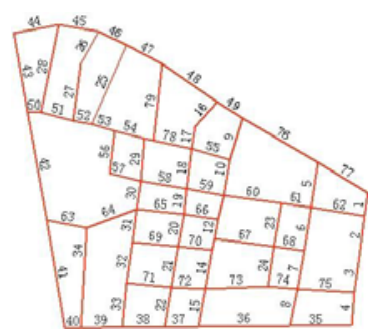

Figure 7. Experimental data

The angular separation threshold is $20^{\circ}$. The $20^{\circ}$ is due to the accumulative effect of street segment. Then the similar orientation graph is formed and its connected components are found. The first component includes street segments: 1, 2, 3, 4, $5,6,7,8,9,10,11,12,13,14,15,16,17,18,19,20,21,22,23$, $24,25,26,27,28,29,30,31,32,33,34,41,42,43,56,79$. The maximal complete subgraph includes street segments: $1,2,3$, 4, $5,6,7,8,9,10,11,12,13,14,15,17,18,19,20,21,22,23,24$, $27,28,29,30,31,32,33,34,56,79$. The corresponding street segment is shown in Figure 8.

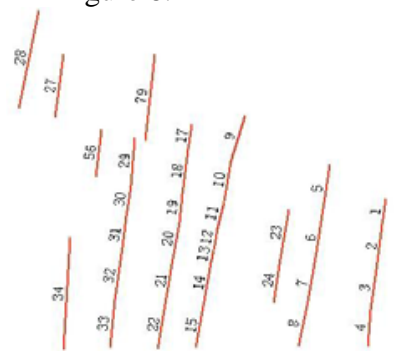

Figure 8. Street segments corresponding to first maximal complete subgraph

The second component includes street segments: 35, 36, 37, 38, $39,40,44,45,46,47,48,49,50,51,52,53,54,55,57,58,59$, $60,61,62,63,64,65,66,67,68,69,70,71,72,73,74,75,76$, 77,78.The maximal complete subgraph includes street segments:35, 36, 37, 38, 39, 40, 45, 50, 51, 52, 53, 54, 55, 57, $58,59,60,61,62,63,65,66,67,68,69,70,71,72,73,74,75$, 78.The corresponding street segment is shown in Figure 9.

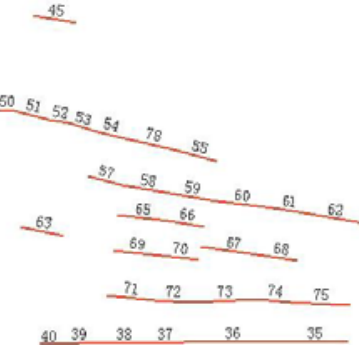

Figure 9. Street segments corresponding to second maximal complete subgraph

We next "Join" the two maximal complete subgraph, then "intersect" with street segment intersection graph created from experimental data in Figure 7. The result is shown in Figure10.

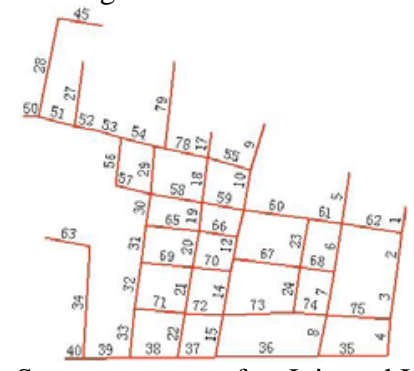

Figure 10. Street segments after Join and Intersection
A candidate is produced after deleting bridges and isolated points until there are no bridges and isolated vertices, as show in Figure 11.

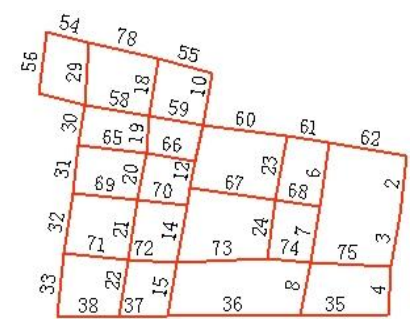

Figure 11. A Candidate

The candidate is processed according to "good continuation" rule to form entire streets, as show in Figure12 (a).The street intersection graph is created (Figure12 (b), and clustering coefficient of this graph is 0 .The candidate is written as final result.

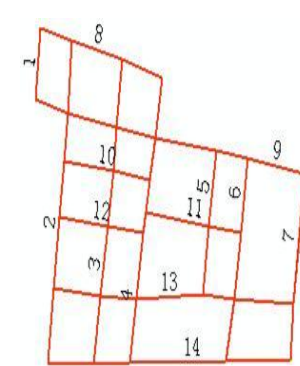

(a)

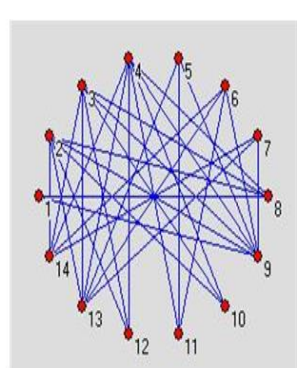

(b)
Figure 12. (a)Entire streets and (b)street intersection relation graph (visualization by Pajek)

\subsection{Discussion}

The experimental results show the proposed approach is valid in detecting the grid pattern in regular and lower degradation situation. The advantages of the proposed approach are: (1)It uses graph-theoretic operations to represent the human recognition knowledge. i.e. finding a set of roughly parallel streets, which are crossed by a second set of parallel streets with roughly perpendicular angle. (2)The only parameter is angular separation threshold. In principle, the parameter is set less than $10^{\circ}$ in regular case and is set less than $20^{\circ}$ in lower degradation cases, which is perceptually satisfactory.

The disadvantages and limitations are: (1) It is incapable of distinguishing the different grid. The approach proposed by Heinzle et al (2005) can do this task; (2)The proposed approach requires the initial street network data must be composed of straight line segments. Therefore, we digitize the data into line segments. (3) Cases similar to Figure13 will be accepted as recognition result by the approach. In fact, these cases are bad results. It should define other evaluation method to exclude these exception cases.

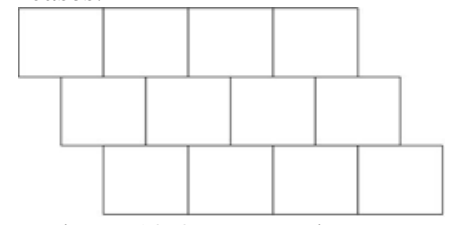

Figure 13 One exception case

\section{CONCLUSIONS AND FUTURE WORK}

This study is significant for answering the key questions in map generalization on the one hand and extends the research field in 
pattern recognition on the other hand. This paper presents a graph based approach to recognition of grid in street networks. This approach takes the geometric features as necessary condition. Through the graph-theoretic operations such as finding connected component, finding maximal complete subgraph, intersection and join, the candidates are produced. Finally, the street intersection graph is formed and clustering coefficient is computed. The experiments illustrates that this graph based approach can be used as an effective method for recognition of grid patterns. Compared with the point-polygon based structural recognition approach, this one is line base structural recognition approach.

There are some issues deserves further research. (1) The experiment is only conducted on simple data. Experiments on more complex data need to be conducted. The evaluation measures to exclude the exception case need to be developed.(2) The comparison with other method to solve this problem need to be done to find the best method to a specific kind of cases.(3) Recognition methods for other patterns in street network need to be developed.

\section{ACKNOWLEDGEMENT}

This research is supported by the National High-Tech Research and Development Plan of China under the grant No.2007AA12Z209, and the National Science Foundation, China under the grant No. 40971242. Special thanks go to an anonymous reviewer for constructive comments that substantially improve quality of the paper.

\section{REFERENCES}

Batagelj, V, Mrvar, A. 2008. Pajek: Program for Analysis and Visualization of Large Networks. Version 1.22.

Diestel, R. Graph Theory (3rd Edition). 2006. Berlin, SpringerVerlag.

Heinzle, F, Ander. K. H, Sester, M. 2005. Graph Based Approaches for Recognition of Patterns and Implicit Information in Road Networks. In: Proceedings of 22nd International Cartographic Conference, A Coruna, Spain.

Heinzle, F., Ander, K. H., Sester, M. 2006. Pattern Recognition in Road Networks on the Example of Circular Road Detection. In: Raubal.M, Miller.H.J, Frank.A.U, Goodchild.M.F.(Eds): Geographic Information Science, GIScience2006, Munster, Germany, LNCS, vol. 4197, pp.253-267.

Heinzle, F., Ander, K. H. 2007. Characterising Space via Pattern Recognition Techniques: Identifying Patterns in Road Networks. In: Mackaness W A, Ruas A, Sarjakoski L T(Eds): Generalisation of Geographic Information: Cartographic Modelling and Applications, Elsevier, pp.233-253.

Iglin, S. 2003. grTheory-Graph Theory Toolbox, http://www.mathworks.com/matlabcentral/fileexchange/4266 (accessed 30 Sep. 2009).

Ip, H., Wong, W. H. 1997. Detecting Perceptually Parallel Curves: Criteria and Force-Driven Optimization. Computer Vision and Image Understanding, 68(2), pp.190-208.
Jiang, B., Claramunt, C. 2004. A Structural Approach to the Model Generalization of urban Street Network. GeoInformatica, 8(2), pp.157-173.

Mackaness, W., Beard, K. 1993. Use of Graph Theory to Support Map Generalization. Cartography and Geographic Information Systems, 20(4), pp.210-221.

Mackaness, W., Edwards, G. 2002. The Importance of Modelling Pattern and Structures in Automated Map Generalization. In: Joint ISPRS/ICA workshop Multi-Scale Representation of Spatial Data, Ottawa, Canada.

Mashall, S. 2005. Streets and Patterns. New York: Spon Press.

Muller, J. C., Weibel R, Lagrange J P, Salg é F. 1995. Generalization: state of the art and issues. In: Muller $\mathrm{J} \mathrm{C}$, Lagrange J P, Weibel R (Eds), GIS and generalization: Methodology and practice. London, Taylor \& Francis, pp.3-17.

Porta, S., Crucitti, P., Latora, V. 2006. The network analysis of urban streets: A dual approach. Physica A, 369, pp.853-866.

Sarkar, S., Boyer, L. 1994. A computational Structure for Preattentive Perceptual Organization: Graphical Enumeration and Voting Methods. IEEE Transactions on man and cybernetics, 24(2), pp.246-266.

Theodoridis, S, Koutroumbas, K. 2009. Pattern Recognition (4th Edition). Amsterdam, Elsevier.

Thomson, R. C., Richardson, D. E. 1999. The "Good Continuation” Principle of Perceptual Organization Applied to the Generalization of Road Networks. In: Proceedings of 19th International Cartographic Conference, Ottawa.

Wang, J.Y. etal. 1993. Principles of Cartographic Generalization. Beijing, Surveying and Mapping Press.

Watts, D. J., Strogatz, S. H. 1998. Collective dynamics of 'small-world' networks. Nature, 393(4), pp.440-442.

Xie, F., Levinson, D. 2007. Measuring the Structure of Road Networks. Geographical Analysis, 39, pp.336-356.

Yang, B. S., Luan, X. C., Li, Q. Q. 2010. An adaptive method for identifying the spatial patterns in road networks. Computers, Environment and Urban Systems, 34(1) pp.40-48

Zhang, Q N. 2004. Modeling Structure and Patterns in Road Network Generalization. In: ICA workshop on Generalization and Multiple Representation, Leicester. 